\title{
Relative Length of Long Paths and Cycles in Graphs with Large Degree Sums
}

DEPARTMENT OF MATHEMATICS,

KEIO UNIVERSITY, HIYOSHI 3-14-1

KOHOKU-KU, YOKOHAMA

KANAGAWA 223, JAPAN

Jan van den Heuvel

FACULTY OF APPLIED MATHEMATICS UNIVERSITY OF TWENTE, P.O. BOX 217 7500 AE ENSCHEDE THE NETHERLANDS

Atsushi Kaneko DEPARTMENT OF ELECTRONIC ENGINEERING KOGAKUIN UNIVERSITY, NISHI-SHINJUKU 1-24-2

SHINJUKU-KU, TOKYO 160, JAPAN

Akira Saito

DEPARTMENT OF MATHEMATICS NIHON UNIVERSITY, SAKURAJOSUI 3-25-40

SETAGAYA-KU, TOKYO 156, JAPAN

\section{ABSTRACT}

For a graph $G, p(G)$ denotes the order of a longest path in $G$ and $c(G)$ the order of a longest cycle. We show that if $G$ is a connected graph on $n \geq 3$ vertices such that $d(u)+d(v)+d(w) \geq n$ for all triples $u, v, W$ of independent vertices, then $G$ satisfies $c(G) \geq p(G)-1$, or $G$ is in one of six families of exceptional graphs. This generalizes results of Bondy and of Bauer, Morgana, Schmeichel, and Veldman. (c) 1995 John Wiley \& Sons, Inc.

\footnotetext{
*Present address: Department of Mathematics and Statistics, Simon Fraser University, Burnaby, B.C., Canada V5A 1 S6.

Journal of Graph Theory, Vol. 20, No. 2, 213-225 (1995)

(C) 1995 John Wiley \& Sons, Inc.

CCC 0364-9024/95/02013-13
} 


\section{INTRODUCTION}

We use Bondy and Murty [4] for terminology and notation not defined here and consider only finite undirected graphs with no loops or multiple edges.

For a graph $G$ and an integer $k$ with $1 \leq k \leq \alpha(G)$, define $\sigma_{k}(G)$ by

$$
\sigma_{k}(G)=\min \left\{\sum_{v \in S} d(v) \mid S \subseteq V(G) \text { an independent set, }|S|=k\right\}
$$

For $k>\alpha(G)$ we set $\sigma_{k}(G)=k(|V(G)|-\alpha(G))$.

$G$ is called 1 -tough if $|S| \geq \omega(G-S)$ for every subset $S \subseteq V(G)$ with $\omega(G-S)>1$, where $\omega(H)$ denotes the number of components of a graph $H$. We use "+" to denote the disjoint union of graphs and $G[S]$ to denote the subgraph of $G$ induced by a nonempty set $S \subseteq V(G)$.

A cycle $C$ of $G$ is called a dominating cycle if every edge of $G$ has at least one of its end vertices on $C$, or, equivalently, if $G-V(C)$ contains no edges. The order of a longest path and a longest cycle in $G$ is denoted by $p(G)$ and $c(G)$, respectively.

There are now several results in graph theory that relate degree sums to the structure of long cycles. Two such results are the following.

Theorem 1 (Bondy [3]). Let $G$ be a 2-connected graph on $n$ vertices such that $\sigma_{3}(G) \geq n+2$. Then every longest cycle in $G$ is a dominating cycle.

Theorem 2 (Bauer, Morgana, Schmeichel, and Veldman [1]). Let $G$ be a 1 -tough graph on $n \geq 3$ vertices such that $\sigma_{3}(G) \geq n$. Then every longest cycle in $G$ is a dominating cycle.

Our main results were inspired by the following easy observation.

Lemma 3. Let $G$ be a connected graph that satisfies $c(G) \geq p(G)-1$. Then every longest cycle in $G$ is a dominating cycle.

Proof. Let $G$ be a connected graph such that $c(G) \geq p(G)-1$ and let $C$ be a longest cycle in $G$. Suppose $G-C$ contains a component $H$ with $|V(H)| \geq 2$. Now it is easy to construct a path in $G$ that contains all vertices on the cycle and at least 2 vertices of $H$, hence contradicting $p(G) \leq c(G)+1$.

Our first result is the next theorem, the proof of which will be given in Section 2.

Theorem 4. Let $G$ be a connected graph on $n$ vertices such that $\sigma_{3}(G) \geq$ $n$. Then $G$ contains a Hamilton path, or $c(G) \geq p(G)-1$. 
Theorem 4 improves the result in Enomoto, Kaneko, and Tuza [5] that if $G$ is a connected graph on $n$ vertices with $\sigma_{3}(G) \geq n$, then $G$ contains a Hamilton path, or every longest cycle in $G$ is a dominating cycle.

Theorem 4 is best possible in the sense that the condition $\sigma_{3}(G) \geq n$ cannot be relaxed, even under a strong connectivity constraint. To see this we construct two classes of graphs. For integers $a, b, c$ with $a \geq b \geq c \geq 2$, define the graph $G_{a, b, c}$ as the join of $K_{1}$ and $K_{a} \cup K_{b} \cup K_{c}$. Then $G_{a, b, c}$ is connected and satisfies $\sigma_{3}\left(G_{a, b, c}\right)=a+b+c=$ $\left|V\left(G_{a, b, c}\right)\right|-1$. Furthermore, $c\left(G_{a, b, c}\right)=a+1$ and $p\left(G_{a, b, c}\right)=$ $a+b+1$, hence $c\left(G_{a, b, c}\right)=p\left(G_{a, b, c}\right)-b \leq p\left(G_{a, b, c}\right)-2$, but $G_{a, b, c}$ also contains no Hamilton path.

Next define, for an integer $t \geq 1$, the graph $H_{t}$ as the join of $K_{t}$ and $(t+2) K_{2}$. Then $H_{t}$ is $t$-connected and satisfies $\sigma_{3}\left(H_{t}\right)=3 t+3=$ $\left|V\left(H_{t}\right)\right|-1$. Furthermore, a longest path in $H_{t}$ has order $3 t+2$ and a longest cycle in $H_{t}$ has order $3 t$. So $H_{t}$ contains no Hamilton path and also $c\left(H_{t}\right)=p\left(H_{t}\right)-2$.

Now we can state our main result, in which we characterize the connected graphs $G$ on $n$ vertices with $\sigma_{3}(G) \geq n$ that do not satisfy $c(G) \geq p(G)-$ 1 .

Theorem 5. Let $G$ be a connected graph on $n \geq 3$ vertices such that $\sigma_{3}(G) \geq n$. Then $G$ satisfies $c(G) \geq p(G)-1$, or $G \in \mathcal{F}(n)$.

Here $\mathcal{F}(n)$ is the class of graphs defined below. Theorem 5 is an immediate consequence of the following result, the proof of which will be given in Section 2. Theorem 6 gives some more information on the relation between paths and cycles in the graphs satisfying the hypothesis of Theorem 5 .

Theorem 6. Let $G$ be a connected graph on $n \geq 3$ vertices such that $\sigma_{3}(G) \geq n$ and suppose $G \notin \mathcal{F}(n)$. Then for every path $P$ in $G$, there exists a cycle $C$ in $G$ such that $|V(P)-V(C)| \leq 1$.

$\mathcal{F}(n)$ is a class of graphs on $n$ vertices consisting of six subclasses:

$$
\mathcal{F}(n)=\mathcal{F}_{1,1}(n) \cup \mathcal{F}_{1,2}(n) \cup \mathcal{F}_{2,1}(n) \cup \mathcal{F}_{2,2}(n) \cup \mathcal{F}_{2,3}(n) \cup \mathcal{F}_{2,4}(n)
$$

The subclasses $\mathcal{F}_{1,2}(n), \ldots, \mathcal{F}_{2,4}(n)$ are defined as follows.

$$
\begin{aligned}
& \mathcal{F}_{1,1}(n): G \in \mathcal{F}_{1,1}(n) \text { if }|V(G)|=n, \sigma_{3}(G) \geq n, V(G)=A_{1} \cup A_{2} \\
& \text { with } A_{1} \cap A_{2}=\varnothing, G\left[A_{1}\right] \text { and } G\left[A_{2}\right] \text { are hamiltonian or } \\
& \text { isomorphic to } K_{2}, \text { and there exists exactly one edge between } \\
& A_{1} \text { and } A_{2} . \\
& \mathcal{F}_{1,2}(n): G \in \mathcal{F}_{1,2}(n) \text { if }|V(G)|=n, \sigma_{3}(G) \geq n \text {, and } V(G)=A_{1} \cup A_{2} \\
& \text { with } A_{1} \cap A_{2}=\{a\}, G\left[A_{1}\right] \text { and } G\left[A_{2}\right] \text { are both hamiltonian or } \\
& \text { both isomorphic to } K_{2} \text {, and there exists no edge between } A_{1}-\{a\} \\
& \text { and } A_{2}-\{a\} .
\end{aligned}
$$




$$
\begin{aligned}
& \mathcal{F}_{2,1}(n): G \in \mathcal{F}_{2,1}(n) \text { if }|V(G)|=n, \sigma_{3}(G) \geq n, \text { and } G \text { is a } \\
& \text { 2-connected spanning subgraph of the join of } K_{2} \text { and } \\
& K_{a}+K_{b}+K_{c}, \text { with } a, b, c \geq 2(n=a+b+c+2) . \\
& \mathcal{F}_{2,2}(n): G \in \mathcal{F}_{2,2}(n) \text { if }|V(G)|=n, \sigma_{3}(G) \geq n, \text { and } G \text { is a } \\
& \text { 2-connected spanning subgraph of the join of } K_{3} \text { and } a K_{2}+b K_{3}, \\
& \text { with } a, b \geq 0 \text { and } a+b=4(n=2 a+3 b+3,11 \leq n \leq \\
&15) . \\
& \mathcal{F}_{2,3}(n): G \in \mathcal{F}_{2,3}(n) \text { if }|V(G)|=n, \sigma_{3}(G) \geq n, \text { and } G \text { is a } \\
& \text { 2-connected spanning subgraph of the join of } K_{s} \text { and } s K_{2}+K_{3}, \\
& \text { with } s \geq 4(n=3 s+3) \text {. } \\
& \mathcal{F}_{2,4}(n): G \in \mathcal{F}_{2,4}(n) \text { if }|V(G)|=n, \sigma_{3}(G) \geq n, \text { and } G \text { is a } \\
& \text { 2-connected spanning subgraph of the join of } K_{s} \text { and }(s+1) K_{2}, \\
& \text { with } s \geq 4(n=3 s+2) .
\end{aligned}
$$

The graphs in $\mathcal{F}(n)$ are not 1-tough, the graphs in $\mathcal{F}_{1,1}(n) \cup \mathcal{F}_{1,2}(n)$ are not 2-connected, and the graphs in $\mathcal{F}_{2,1}(n) \cup \mathcal{F}_{2,2}(n) \cup \mathcal{F}_{2,3}(n) \cap \mathcal{F}_{2,4}(n)$ are 2 -connected but satisfy $\sigma_{3}(G) \leq n+1$. These observations show that Theorem 5 implies the following results, which are, by Lemma 3 , generalizations of Theorems 1 and 2, respectively.

\section{Corollary 7.}

(a) Let $G$ be a 2-connected graph on $n$ vertices such that $\sigma_{3}(G) \geq n+2$. Then $G$ satisfies $c(G) \geq p(G)-1$.

(b) Let $G$ be a 1-tough graph on $n \geq 3$ vertices such that $\sigma_{3}(G) \geq n$. Then $G$ satisfies $c(G) \geq p(G)-1$.

Theorem 5 also implies the following improvement of Bauer, Morgana, Schmeichel, and Veldman [1, Lemma 8]. The proof of Corollary 8 will be given in Section 2 too.

Corollary 8. Let $G$ be a connected graph on $n \geq 3$ vertices such that $\sigma_{3}(G) \geq n$. Suppose $G$ contains a longest cycle $C$ which is a dominating cycle. Let $A=\bigcup_{v \in V(G)-V(C)} N(v)$. Fix an orientation $\vec{C}$ on $C$ and let $A^{+}$ denote the set of vertices immediately following the vertices of $A$ on $\vec{C}$. Then $(V(G)-V(C)) \cup A^{+}$is an independent set of vertices.

In Van den Heuvel [6] it is shown that the conclusions from Corollary 8 can be extended in order to obtain a version of the Hopping Lemma from Woodall [7] that uses all vertices outside the cycle. Using these results, in [6] several new lower bounds for the lengths of longest cycles in graphs with large degree sums are proved.

\section{PROOFS OF THE RESULTS}

First we introduce some additional notation. 
If $P$ is a path in a graph $G$, then we denote by $\vec{P}$ the path $P$ with a given orientation, and by $\overleftarrow{P}$ the same path with reverse orientation. If $u, v \in V(P)$ and $u$ precedes $v$ on $\vec{P}$, then $u \vec{P} v$ denotes the consecutive vertices of $P$ from $u$ to $v$. The same vertices in reverse order are given by $v \stackrel{P}{P} u$. We will consider $u \vec{P} v$ and $v \overleftarrow{P}_{u}$ both as paths and as vertex sets. If $u \in V(P)$, then $u^{+}$denotes the successor of $u$ on $\vec{P}$ and $u^{-}$its predecessor. For $U \subseteq V(P)$, $U^{+}=\left\{u^{+} \mid u \in U\right\}$ and $U^{-}=\left\{u^{-} \mid u \in U\right\}$. Similar notation is used for cycles.

An extension of $P$ is a path $P^{\prime}$ with $V(P) \subseteq V\left(P^{\prime}\right)$ and $V(P) \neq V\left(P^{\prime}\right) . P$ is called nonextendable if there exists no extension of $P$.

First we prove Theorem 4. It is a consequence of the following result, in the same way as Theorem 5 follows from Theorem 6 .

Theorem 9. Let $G$ be a connected graph on $n \geq 3$ vertices such that $\sigma_{3}(G) \geq n$ and let $P$ be a nonextendable path in $G$. Then $P$ is a Hamilton path, or there exists a cycle $C$ in $G$ such that $|V(P)-V(C)| \leq 1$.

Proof. Let $G$ be a connected graph on $n \geq 3$ vertices with $\sigma_{3}(G) \geq n$. Let $P=x_{1} \vec{P} x_{p}$ be a nonextendable path in $G$. Suppose $P$ is not a Hamilton path and there exists no cycle $C$ in $G$ such that $|V(P)-V(C)| \leq 1$. Since $G$ is connected and $n \geq 3$, we may assume $|V(P)| \geq 3$. Let $y \in$ $V(G)-V(P)$. Since $P$ is nonextendable, we have $N\left(x_{1}\right) \subseteq V(P)-\left\{x_{1}\right\}$ and $N\left(x_{p}\right) \subseteq V(P)-\left\{x_{p}\right\}$. Set

$$
A=N\left(x_{1}\right)^{-}, \quad B=N\left(x_{p}\right)^{+}, \quad \text { and } D=N(y)
$$

If $x \in A \cap D$, then the path $y x \stackrel{\sim}{P} x_{1} x^{+} \vec{P} x_{p}$ is an extension of $P$, contradicting the assumption. Therefore, we have $A \cap D=\varnothing$ and, similarly, $B \cap D=\varnothing$. If $x \in A \cap B$, then $C=x^{-} \stackrel{\leftarrow}{P} x_{1} x^{+} \vec{P} x_{p} x^{-}$is a cycle with $|V(P)-V(C)|=1$, a contradiction. Thus we have $A \cap B=\varnothing$. Finally, if $x_{1} x_{p} \in E(G)$, then $C=x_{1} \vec{P} x_{p} x_{1}$ is a cycle with $V(P)=V(C)$, again a contradiction. So $\left\{x_{1}, x_{p}, y\right\}$ is an independent set and we have, since $y \notin A \cup B \cup D$,

$$
\begin{aligned}
n & \leq \sigma_{3}(G) \leq d\left(x_{1}\right)+d\left(x_{p}\right)+d(y)=\left|A^{+}\right|+\left|B^{-}\right|+|D| \\
& =|A|+|B|+|D|=|A \cup B \cup D| \leq n-1 .
\end{aligned}
$$

This contradiction completes the proof.

The remainder of this section is devoted to the proof of Theorem 6 .

Proof of Theorem 6. Let $G$ be a graph on $n \geq 3$ vertices with $\sigma_{3}(G) \geq$ $n$ and suppose there exists a path $P=x_{1} \vec{P} x_{p}$ in $G$ such that there is no cycle $C$ with $|V(P)-V(C)| \leq 1$. If $P^{\prime}$ is an extension of $P$ and $C^{\prime}$ is a cycle such that $\left|V\left(P^{\prime}\right)-V\left(C^{\prime}\right)\right| \leq 1$, then also $\left|V(P)-V\left(C^{\prime}\right)\right| \leq 1$. So, without 


\section{8}

loss of generality, we may assume that $P$ is nonextendable. By Theorem 9 this means that $P$ is a Hamilton path, hence $p(G)=n$. Since $G$ does not contain a cycle $C$ with $|V(P)-V(C)| \leq 1$, we conclude $c(G) \leq n-2$.

For a Hamilton path $Q=x_{1} \vec{Q} x_{n}=x_{1} x_{2} \ldots x_{n}$, define

$$
r(Q)=\max \left\{i \mid x_{i} \in N\left(x_{1}\right)\right\}
$$

and

$$
s(Q)=\min \left\{j \mid x_{j} \in N\left(x_{n}\right)\right\} .
$$

Now suppose $P=x_{1} \vec{P} x_{n}=x_{1} x_{2} \ldots x_{n}$ is chosen such that

(1) $r(P)$ is as large as possible, and

(2) $s(P)$ is as small as possible, subject to (1).

Let $r=r(P)$ and $s=s(P)$. Since $c(G) \leq n-2$ and $n \geq 3$, we have $x_{1} x_{n} \notin E(G)$.

We consider four cases, depending on the relative values of $r$ and $s$. In each case we obtain a contradiction, or we reach the conclusion $G \in \mathcal{F}(n)$.

Case 1. $r \leq s-2$. Let $y \in x_{r}^{+} \vec{P} x_{s}^{-}$. By the definition of $r$ and $s$ we have $x_{1} y, x_{n} y \notin E(G)$, hence $\left\{x_{1}, x_{n}, y\right\}$ is an independent set. Furthermore, $N\left(x_{1}\right) \subseteq x_{1}^{+} \vec{P} x_{r}$ and $N\left(x_{n}\right) \subseteq x_{s} \vec{P} x_{n}^{-}$. Define

$$
A=N\left(x_{1}\right)^{-}, \quad B=N\left(x_{n}\right)^{+}, \quad \text { and } D=N(y) .
$$

Since $r \leq s-2$, we have $A \cap B=\varnothing$. If $x \in A \cap D$, then the path $P^{\prime}=x \bar{P} x_{1} x^{+} \vec{P} x_{n}$ is a Hamilton path with $r\left(P^{\prime}\right)>r$, contradicting the choice of $P$ in (1), while if $x \in B \cap D$, then the path $x_{1} \vec{P} x^{-} x_{n} \breve{P} x$ is a path that contradicts the choice of $P$ in (2). So we have $A \cap D=B \cap D=\varnothing$. Since $\left\{x_{1}, x_{n}, y\right\}$ is an independent set and $y \notin A \cup B \cup D$, we reach a contradiction as in the proof of Theorem 9 .

Case 2. $r=s-1$. If $x_{r} x_{s}$ is a cut edge, then $G \in \mathcal{F}_{1,1}(n)$. So we can assume there exists an edge $y_{1} y_{2}$ with $y_{1} \in x_{1} \vec{P} x_{r}^{-}$and $y_{2} \in x_{s} \vec{P} x_{n}$, or $y_{1} \in x_{1} \vec{P} x_{r}$ and $y_{2} \in x_{s}^{+} \vec{P} x_{n}$. First suppose $y_{1} \in x_{1} \vec{P} x_{r}^{-}$and $y_{2} \in x_{s} \vec{P} x_{n}$. Then $x_{1} y_{1}^{+} \notin E(G)$, otherwise the path $y_{1} \check{P} x_{1} y_{1}^{+} \vec{P} x_{n}$ contradicts the choice of $P$ in (1); and $x_{n} y_{1}^{+} \notin E(G)$, by the definition of $s$. So $\left\{x_{1}, x_{n}, y_{1}^{+}\right\}$is an independent set. Set

$$
\begin{array}{ll}
A=N\left(x_{1}\right), & D_{1}=\left(N\left(y_{1}^{+}\right) \cap x_{1} \vec{P} y_{1}\right)^{+}, \\
B=N\left(x_{n}\right), & D_{2}=\left(N\left(y_{1}^{+}\right) \cap y_{1}^{++} \vec{P} x_{n}\right)^{-} .
\end{array}
$$


Since $N\left(x_{1}\right) \subseteq x_{1}^{+} \vec{P} x_{r}, N\left(x_{n}\right) \subseteq x_{s} \vec{P} x_{n}^{-}, r=s-1$, and $y_{1}^{+} \in x_{1} \vec{P} x_{r}$, we have $A \cap B=\varnothing$ and $B \cap D_{1}=\varnothing$. Furthermore, $D_{1} \cap D_{2}=\left\{y_{1}^{+}\right\}$. If $x \in A \cap D_{1}$, then the path $y_{1} \stackrel{P}{P} x x_{1} \vec{P} x^{-} y_{1}^{+} \vec{P} x_{n}$ contradicts the choice of $P$ in (1); and if $x \in A \cap D_{2}$, then $y_{1} \tilde{P} x_{1} x \vec{P} y_{1}^{+} x^{+} \vec{P} x_{n}$ contradicts the choice of $P$ in (1). This gives $A \cap D_{1}=A \cap D_{2}=\varnothing$. Finally, if $x \in$ $B \cap D_{2}$, then the path $P^{\prime}=x_{1} \vec{P} x x_{n} \vec{P} x^{+}$is a Hamilton path with $r\left(P^{\prime}\right)=r$ and $s\left(P^{\prime}\right)<s$, contradicting the choice of $P$ in (2). So $B \cap D_{2}=\varnothing$. This shows $|A|+|B|+\left|D_{1}\right|+\left|D_{2}\right|=\left|A \cup B \cup D_{1} \cup D_{2}\right|+1$. Also, $x_{1}, x_{n} \notin A \cup B \cup D_{1} \cup D_{2}$, hence it follows that

$$
\begin{aligned}
n & \leq \sigma_{3}(G) \leq d\left(x_{1}\right)+d\left(x_{n}\right)+d\left(y_{1}^{+}\right)=|A|+|B|+\left|D_{1}^{-}\right|+\left|D_{2}^{+}\right| \\
& =|A|+|B|+\left|D_{1}\right|+\left|D_{2}\right|=\left|A \cup B \cup D_{1} \cup D_{2}\right|+1 \\
& \leq n-2+1=n-1,
\end{aligned}
$$

a contradiction.

If $y_{1} \in x_{1} \vec{P} x_{r}$ and $y_{2} \in x_{s}^{+} \vec{P} x_{n}$, then in a similar way a contradiction is reached by considering the vertices $x_{1}, x_{n}$, and $y_{2}^{-}$.

Case 3. $r=s$. If $x_{r}\left(=x_{s}\right)$ is a cut vertex, then $G \in \mathcal{F}_{1,2}(n)$. So we can assume there exists an edge $y_{1} y_{2}$ with $y_{1} \in x_{1} \vec{P} x_{r}^{-}$and $y_{2} \in x_{s}^{+} \vec{P} x_{n}$. Assume $y_{1}, y_{2}$ are chosen such that $\left|y_{1} \vec{P} y_{2}\right|$ is minimum. Then $x_{1} y_{1}^{+} \notin$ $E(G)$, otherwise the path $y_{1} \stackrel{P}{P} x_{1} y_{1}^{+} \vec{P} x_{n}$ contradicts the choice of $P$ in (1), and, similarly, $x_{n} y_{2}^{-} \notin E(G)$. This also shows that $y_{1}^{+} \neq x_{r}$ and $y_{2}^{-} \neq x_{r}$. Furthermore, $x_{n} y_{1}^{+} \notin E(G)$, by the definition of $s$. So $\left\{x_{1}, x_{n}, y_{1}^{+}\right\}$is an independent set. Define

$$
\begin{array}{ll}
A=N\left(x_{1}\right), & D_{1}=\left(N\left(y_{1}^{+}\right) \cap x_{1} \vec{P} y_{1}\right)^{+}, \\
B=N\left(x_{n}\right), & D_{2}=\left(N\left(y_{1}^{+}\right) \cap y_{1}^{++} \vec{P} x_{n}\right)^{-} .
\end{array}
$$

Since $N\left(x_{1}\right) \subseteq x_{1}^{+} \vec{P} x_{r}, N\left(x_{n}\right) \subseteq x_{s} \vec{P} x_{n}^{-}$, and $r=s$, we have $|A \cap B|=1$. Similar to Case 2 we can prove $D_{1} \cap D_{2}=\left\{y_{1}^{+}\right\}$and $A \cap D_{1}=A \cap$ $D_{2}=B \cap D_{1}=B \cap D_{2}=\varnothing$. This shows $|A|+|B|+\left|D_{1}\right|+\left|D_{2}\right|=$ $\left|A \cup B \cup D_{1} \cup D_{2}\right|+2$. Since $y_{2}^{-} \in x_{r}^{+} \vec{P} x_{n}$ and $x_{n} y_{2}^{-} \notin E(G)$, we have $y_{2}^{-} \notin A \cup B \cup D_{1}$. Finally, we have $y_{1}^{+} y_{2} \notin E(G)$, by the choice of $y_{1}$ and $y_{2}$, so $y_{2}^{-} \notin D_{2}$. Thus we have $x_{1}, x_{n}, y_{2}^{-} \notin A \cup B \cup D_{1} \cup D_{2}$. If follows that

$$
\begin{aligned}
n & \leq \sigma_{3}(G) \leq d\left(x_{1}\right)+d\left(x_{n}\right)+d\left(y_{1}^{+}\right)=|A|+|B|+\left|D_{1}^{-}\right|+\left|D_{2}^{+}\right| \\
& =|A|+|B|+\left|D_{1}\right|+\left|D_{2}\right|=\left|A \cup B \cup D_{1} \cup D_{2}\right|+2 \\
& \leq n-3+2=n-1,
\end{aligned}
$$

the final contradiction in this case. 
Case 4. $r \geq s+1$. In this case we know that $G$ is 2-connected. Let $H$ be the path $x_{r}^{+} \vec{P} x_{n}$. By the maximality of $r$ we have $N\left(x_{1}\right) \cap V(H)=\varnothing$.

Let $Q$ be the path $x_{s}^{+} \vec{P} x_{r} x_{1} \vec{P} x_{s} x_{n} \dot{P} x_{r}^{+}$. Then the path $Q$ satisfies $r(Q) \geq$ $r$, hence $r(Q)=r$, and $r(Q) \geq s(Q)+1$. And we also have $N\left(x_{s}^{+}\right) \cap$ $V(H)=\varnothing$. Note that the path $Q$ satisfies (1), but does not necessarily satisfy (2). In the remainder of the proof we often reach a contradiction by the construction of a path that contradicts the choice of $P$ in (1). In these cases the path $Q$ can play a similar role as the path $P$.

For $x \in V(H)$, let $A_{x}=N(x) \cap x_{1} \vec{P} x_{r}$, and set $A=\bigcup_{x \in V(H)} A_{x}$.

Claim 1. If $a \in A$, then $a^{+} \notin N\left(x_{1}\right) \cup N\left(x_{s}^{+}\right)$.

Proof of Claim 1. Suppose $a \in A_{x}$ with $a^{+} \in N\left(x_{1}\right) \cup N\left(x_{s}^{+}\right)$for some $x \in V(H)$. First suppose $a \in x_{1} \vec{P} x_{s}$. Assume $a=x_{s}$. Then $a^{+}=x_{s}^{+}$ and $a^{+} \notin N\left(x_{s}^{+}\right)$. If $x_{s}^{+}=a^{+} \in N\left(x_{1}\right)$, then $x_{1} \vec{P} x_{s} x_{n} \stackrel{P}{P} x_{s}^{+} x_{1}$ is a Hamilton cycle, a contradiction. Hence we may assume $a \neq x_{s}$, thus $\left\{a, a^{+}\right\} \subseteq x_{1} \vec{P} x_{s}$. If $a^{+} \in N\left(x_{1}\right)$, then the path $a \bar{P} x_{1} a^{+} \vec{P} x_{n}$ contradicts the choice of $P$ in (1). If $a^{+} \in N\left(x_{s}^{+}\right)$, then the path $x_{1} \vec{P} a x \vec{P} x_{n} x_{s} \stackrel{t}{P} a^{+} x_{s}^{+} \vec{P} x^{-}$contradicts the choice of $P$ in (1). We conclude $a^{+} \notin N\left(x_{1}\right) \cup N\left(x_{s}^{+}\right)$.

If $a \in x_{s}^{+} \vec{P} x_{r}$, then we reach the same conclusion by considering the path $Q$ instead of $P$.

Now set

$$
\begin{aligned}
& R_{1}=\left(N\left(x_{1}\right) \cap x_{1} \vec{P} x_{s}\right)^{-}, \quad S_{1}=N\left(x_{s}^{+}\right) \cap x_{1} \vec{P} x_{s}, \\
& R_{2}=N\left(x_{1}\right) \cap x_{s}^{+} \vec{P} x_{r}, \quad S_{2}=\left(N\left(x_{s}^{+}\right) \cap x_{s}^{+} \vec{P} x_{r}\right)^{-},
\end{aligned}
$$

and

$$
R=R_{1} \cup R_{2}, \quad S=S_{1} \cup S_{2} .
$$

Claim 2. $d\left(x_{1}\right)=|R|$ and $d\left(x_{s}^{+}\right)=|S|$.

Proof of Claim 2. We have $N\left(x_{1}\right)=R_{1}^{+} \cup R_{2}$ and $R_{1}^{+} \cap R_{2}=\varnothing$. So $d\left(x_{1}\right)=\left|R_{1}^{+}\right|+\left|R_{2}\right|=\left|R_{1}\right|+\left|R_{2}\right|=|R|$.

The claim $d\left(x_{s}^{+}\right)=|S|$ is proved in the same way.

Claim 3. $R \cap S=\varnothing$.

Proof of Claim 3. Assume $R \cap S \neq \varnothing$ and let $a \in R \cap S$. First suppose $a \in x_{1} \vec{P} x_{s}$. By definition, $x_{s} \notin R$, so $\left\{a, a^{+}\right\} \subseteq x_{1} \vec{P} x_{s}$. This means $a \in R_{1} \cap S_{1}$, so $a^{+} \in N\left(x_{1}\right)$ and $a \in N\left(x_{s}^{+}\right)$. Then $x_{1} \vec{P} a x_{s}^{+} \vec{P} x_{n} x_{s} \stackrel{P}{P} a^{+} x_{1}$ is a Hamilton cycle, a contradiction. 
If $a \in x_{s}^{+} \vec{P} x_{r}$, then the same conclusion is obtained by considering the path $Q$.

Claim 4. If $a \in A-\left\{x_{r}, x_{s}\right\}$, then $\left\{a, a^{+}\right\} \nsubseteq R \cup S$.

Proof of Claim 4. Let $a \in A-\left\{x_{r}, x_{s}\right\}$ and assume $\left\{a, a^{+}\right\} \subseteq R \cup S$. Again, we only consider the case $a \in x_{1} \vec{P} x_{s}$; the case $a \in x_{s}^{+} \vec{P} x_{r}$ is proved similarly by considering the path $Q$. Since $a \neq x_{s}$, $\left\{a, a^{+}\right\} \subseteq x_{1} \vec{P} x_{s}$, hence $\left\{a, a^{+}\right\} \subseteq R_{1} \cup S_{1}$. By Claim 1, $a \notin R_{1}$ and $a^{+} \notin S_{1}$. Therefore, we have $a \in S_{1}$ and $a^{+} \in R_{1}$. This means $a \in N\left(x_{s}^{+}\right)$and $a^{++} \in N\left(x_{1}\right) \cap x_{1} \vec{P} x_{s}$. We can construct the cycle $x_{1} \vec{P} a x_{s}^{+} \vec{P} x_{n} x_{s} \stackrel{\leftarrow}{P} a^{++} x_{1}$ of length $n-1$, a contradiction.

Claim 5. $A_{x_{n}}^{+} \cap A=\varnothing, A_{x_{n}}^{-} \cap A=\varnothing, A_{x_{r}^{+}}^{+} \cap A=\varnothing$, and $A_{x_{r}^{+}}^{-} \cap A=$ $\varnothing$.

Proof of Claim 5. First we assume $A_{x_{n}}^{+} \cap A \neq \varnothing$, say $a^{+} \in A_{x_{n}}^{+} \cap A$. Then $a \in N\left(x_{n}\right) \cap x_{1} \vec{P} x_{r}$ and $a^{+} \in N(x) \cap x_{1} \vec{P} x_{r}$ for some $x \in V(H)$. This implies $a^{+} \neq x_{1}$. The path $x_{1} \vec{P} a x_{n} \vec{P} x a^{+} \vec{P} x^{-}$contradicts the choice of $P$ in (1).

Next, assume $A_{x_{n}}^{-} \cap A \neq \varnothing$, say $a^{-} \in A_{x_{n}}^{-} \cap A$. Then $a \in N\left(x_{n}\right) \cap$ $x_{1} \vec{P} x_{r}$ and $a^{-} \in N(x) \cap x_{1} \vec{P} x_{r}$ for some $x \in V(H)$. This implies $a \neq x_{1}$, and the path $x_{1} \vec{P} a^{-} x \vec{P} x_{n} a \vec{P} x^{-}$contradicts the choice of $P$ in (1) again.

We can prove $A_{x_{r}^{+}}^{+} \cap A=\varnothing$ and $A_{x_{r}^{+}}^{-} \cap A=\varnothing$ in the same way, by considering the path $Q$ instead of $P$

We call a vertex $x \in V(H)$ good if $A_{x}^{+} \cap A_{x}=\varnothing$. Then $x_{n}$ is a good vertex since $A_{x_{n}}^{+} \cap A_{x_{n}} \subseteq A_{x_{n}}^{+} \cap A=\varnothing$.

Suppose $x \in V(H)$ is a good vertex. Let $A_{x}-\left\{x_{r}, x_{s}\right\}=\left\{a_{1}, \ldots, a_{k(x)}\right\}$. For $i=1, \ldots, k(x)$, there exists a vertex $b_{i} \in\left\{a_{i}, a_{i}^{+}\right\}$such that $b_{i} \notin$ $R \cup S$, by Claim 4. Since $A_{x}^{+} \cap A_{x}=\varnothing$, we have $b_{i} \neq b_{j}$ if $i \neq j$. Let

$$
\ell(x)=\left|x_{1} \vec{P} x_{r}-(R \cup S)-\left\{b_{1}, \ldots, b_{k(x)}\right\}\right| .
$$

Then $|R \cup S|=r-k(x)-\ell(x)$. Let $\delta(x)=\left|A_{x} \cap\left\{x_{r}, x_{s}\right\}\right|$. Then we have $0 \leq \delta(x) \leq 2$ and $\left|A_{x}\right|=k(x)+\delta(x)$. Therefore,

$$
k(x)=\left|A_{x}\right|-\delta(x)=d_{G}(x)-d_{H}(x)-\delta(x) .
$$

Let $\quad \alpha(x)=|V(H)|-1-d_{H}(x)=n-r-1-d_{H}(x) \quad(0 \leq \alpha(x) \leq$ $n-r-1)$. Then $k(x)=d_{G}(x)-n+r+1+\alpha(x)-\delta(x)$, and so 
we have

$$
\begin{aligned}
|R \cup S| & =r-\ell(x)-d_{G}(x)+n-r-1-\alpha(x)+\delta(x) \\
& =n-d_{G}(x)-\ell(x)-1-\alpha(x)+\delta(x) .
\end{aligned}
$$

On the other hand, $|R \cup S|=|R|+|S|=d\left(x_{1}\right)+d\left(x_{s}^{+}\right)$, by Claims 2 and 3 , and this means

$$
d\left(x_{1}\right)+d\left(x_{s}^{+}\right)+d(x)=n-\ell(x)-1-\alpha(x)+\delta(x) .
$$

Since $\sigma_{3}(G) \geq n$ and $\left\{x_{1}, x_{s}^{+}, x\right\}$ is an independent set, we have $n-\ell(x)-$ $1-\alpha(x)+\delta(x) \geq n$, or $\delta(x) \geq 1+\alpha(x)+\ell(x)$. Thus we have proved the following claim.

Claim 6. If $x \in V(H)$ is a good vertex, then $2 \geq \delta(x) \geq 1+\alpha(x)+$ $\ell(x)$. In particular, $2 \geq \delta\left(x_{n}\right) \geq 1+\alpha\left(x_{n}\right)+\ell\left(x_{n}\right)$

Claim 7. $A \cap A^{+}=\varnothing$.

Proof of Claim 7. Assume $A \cap A^{+} \neq \varnothing$, say $a^{+} \in A \cap A^{+}$. First suppose $a \in x_{1} \vec{P} x_{s}$. Let $a \in A_{x}$ and $a^{+} \in A_{y}$ for $x, y \in V(H)$. By Claim 5, $x, y \neq x_{n}$. We consider two cases.

Case 7.1. $y \in x \vec{P} x_{n}$. If $x^{-} \in N\left(x_{n}\right)$, then the existence of the path $x_{1} \vec{P}$ ax $\vec{P}$ ya $a^{+} \vec{P} x^{-} x_{n} \stackrel{\leftrightarrow}{P} y^{+}$contradicts the choice of $P$ in (1). Therefore, we have $x^{-} \notin N\left(x_{n}\right)$. If $x \neq x_{r}^{+}$, then $\alpha\left(x_{n}\right) \geq 1$. This implies $\alpha\left(x_{n}\right)=1$, $\ell\left(x_{n}\right)=0$, and $\delta\left(x_{n}\right)=2$, by Claim 6. If $x=x_{r}^{+}$, then $x_{r}=x^{-} \in N\left(x_{n}\right)$, hence we have $\delta\left(x_{n}\right) \leq 1$.. This implies $\delta\left(x_{n}\right)=1$ and $\alpha\left(x_{n}\right)=\ell\left(x_{n}\right)=0$, again by Claim 6 . Therefore, we have $\ell\left(x_{n}\right)=0$ in either case. By Claim 5, $\left\{a, a^{+}\right\} \cap\left(A_{x_{n}}^{+} \cup A_{x_{n}}\right)=\varnothing$. Since $\ell\left(x_{n}\right)=0$, this implies $\left\{a, a^{+}\right\} \subseteq R \cup S$. By Claim 4, this means $a \notin A-\left\{x_{r}, x_{s}\right\}$, hence $a=x_{s}$. So $a^{+} \in A_{x_{n}}^{+} \cap A$, which contradicts Claim 5 .

Case 7.2. $x \in y^{+} \vec{P} x_{n}$. If $y^{-} \in N\left(x_{n}\right)$, then the path $x_{1} \vec{P} a x$ $\stackrel{\leftarrow}{P} y a^{+} \vec{P} y^{-} x_{n} \stackrel{P}{P} x^{+}$contradicts the choice of $P$ in (1). Hence we have $y^{-} \notin N\left(x_{n}\right)$. This implies $\ell\left(x_{n}\right)=0$, and by the same argument as in Case 7.1 we obtain a contradiction.

If $a \in x_{s}^{+} \vec{P} x_{r}$, we consider the path $Q$ to reach similar contradictions.

By Claim 7, all vertices in $H$ are good.

Claim 8. $\left|A-A_{x_{n}}-\left\{x_{r}, x_{s}\right\}\right| \leq 1$ and $\left|A-A_{x_{r}^{+}}-\left\{x_{r}, x_{s}\right\}\right| \leq 1$. 
Proof of Claim 8. Assume $\left|A-A_{x_{n}}-\left\{x_{r}, x_{s}\right\}\right| \geq 2$, say $a, b \in A-$ $A_{x_{n}}-\left\{x_{r}, x_{s}\right\}, a \neq b$. By Claim 4 we have $\left\{a, a^{+}\right\} \nsubseteq R \cup S$ and $\left\{b, b^{+}\right\} \nsubseteq$ $R \cup S$. Let $a^{\prime} \in\left\{a, a^{+}\right\}$and $b^{\prime} \in\left\{b, b^{+}\right\}$such that $\left\{a^{\prime}, b^{\prime}\right\} \cap(R \cup S)=$ $\varnothing$. By Claim 7 we have $a^{\prime} \neq b^{\prime}$. By Claim 5 and the assumption that $a \notin A_{x_{n}}$ we have $\left\{a, a^{+}\right\} \cap\left(A_{x_{n}}^{+} \cup A_{x_{n}}\right)=\varnothing$, and hence $a^{\prime} \notin A_{x_{n}}^{+} \cup A_{x_{n}}$. This means $a^{\prime} \in x_{1} \vec{P} x_{r}-(R \cup S)-\left(A_{x_{n}}^{+} \cup A_{x_{n}}\right)$. Similarly, we obtain $b^{\prime} \in x_{1} \vec{P} x_{r}-(R \cup S)-\left(A_{x_{n}}^{+} \cup A_{x_{n}}\right)$ and hence $\ell\left(x_{n}\right) \geq 2$. By Claim 6 this gives $\delta\left(x_{n}\right) \geq 1+\alpha\left(x_{n}\right)^{\prime}+\ell\left(x_{n}\right) \geq 3$, a contradiction.

By the same arguments we can show $\left|A-A_{x_{r}^{+}}-\left\{x_{r}, x_{s}\right\}\right| \leq 1$.

Claim 9. For any distinct $a, b \in A$, there exists a Hamilton path $P_{a b}=$ $y_{1} \vec{P}_{a b} y_{n-r}$ in $H$ with $a \in N\left(y_{1}\right)$ and $b \in N\left(y_{n-r}\right)$.

Proof of Claim 9. We consider three cases, one of which is trivial.

Case 9.1. $\{a, b\} \cap\left\{x_{r}, x_{s}\right\}=\varnothing$. By Claim 8, $\{a, b\} \cap A_{x_{n}} \neq \varnothing$ and $\{a, b\} \cap A_{x_{r}^{+}} \neq \varnothing$. We may assume $a \in A_{x_{n}}$. If $b \in A_{x_{r}^{+}}$, then $x_{n} \overleftarrow{P} x_{r}^{+}$ is a required Hamilton path in $H$. So we may assume $\{a, b\} \cap A_{x_{r}^{+}}=\{a\}$ and hence $\{a, b\} \cap A_{x_{n}}=\{a\}$.

Let $b \in A_{x}\left(x \neq x_{r}^{+}, x_{n}\right)$. Since $b \in A-A_{x_{n}}-\left\{x_{r}, x_{s}\right\}$, it follows that $\left\{b, b^{+}\right\} \nsubseteq R \cup S$, by Claim 4 , and $\left\{b, b^{+}\right\} \cap\left(A_{x_{n}}^{+} \cup A_{x_{n}}\right)=\varnothing$. This implies $\ell\left(x_{n}\right) \geq 1$. Therefore, we have $\delta\left(x_{n}\right)=2, \alpha\left(x_{n}\right)=0$, and $\ell\left(x_{n}\right)=1$ by Claim 6. Since $\alpha\left(x_{n}\right)=0$, we have $x^{-} \in N\left(x_{n}\right)$. Then $x_{r}^{+} \vec{P} x^{-} x_{n} \stackrel{\leftarrow}{P} x$ is a required Hamilton path in $H$.

Case 9.2. $\left\{\{a, b\} \cap\left\{x_{r}, x_{s}\right\} \mid=1\right.$. We may assume $a=x_{r}$ and $b \neq x_{s}$. If $b \in A_{x_{n}}$, then $x_{r}^{+} \vec{P} x_{n}$ is a required Hamilton path. Thus we may assume $b \notin A_{x_{n}}$. Then we have $\ell\left(x_{n}\right) \geq 1$ and hence $\alpha\left(x_{n}\right)=0$ and $\delta\left(x_{n}\right)=2$. Let $b \in A_{x}-\left\{x_{r}, x_{s}\right\}$. If $x \neq x_{r}^{+}$, then $x^{-} \in N\left(x_{n}\right)$ since $\alpha\left(x_{n}\right)=0$, and $x_{r}^{+} \vec{P} x^{-} x_{n} \stackrel{P}{P} x$ is a required Hamilton path. If $x=x_{r}^{+}$, then we have $a=x_{r} \in N\left(x_{n}\right)$ since $\delta\left(x_{n}\right)=2$, and $x_{n} \widetilde{P} x_{r}^{+}$is a required path.

Case 9.3. $\{a, b\}=\left\{x_{r}, x_{s}\right\}$. This case is trivial.

This completes the proof of Claim 9 .

By Claim 9 we can describe the structure of $G$ as follows: $G$ contains a cycle $C=x_{1} \vec{P} x_{r} x_{1}$ and a path $H=x_{r}^{+} \vec{P} x_{n}$ satisfying $V(H)=G-V(C)$ and $|V(H)| \geq 2$, such that, if we set $A=N_{G}(H)$, then for any distinct $a, b \in A$, there exists a Hamilton path $P_{a b}=y_{1} \vec{P}_{a b} y_{n-r}$ in $H$ with $a \in N\left(y_{1}\right)$ and $b \in N\left(y_{n-r}\right)$. This means that we can use the following result, established implicitly in the proof of Bauer et al. [1, Theorem 5] (already cited in Theorem 2). 
Theorem 10. (Bauer et al. [1]). Let $G$ be a 2-connected graph on $n$ vertices with $\sigma_{3}(G) \geq n$. Suppose $G$ contains a cycle $C$ and a nontrivial component $H$ (i.e., $|V(H)| \geq 2$ ) of $G-V(C)$. Then one of the following holds.

(a) there is a cycle $C^{\prime}$ in $G$ such that $\left|V\left(C^{\prime}\right) \cap V(H)\right| \geq 1, \mid V\left(C^{\prime}\right) \cap$ $V(C)|\geq| V(C) \mid-1$, and the vertices in $V\left(C^{\prime}\right) \cap V(H)$ form a path in $C^{\prime}$, or

(b) there exists a nonempty subset $S \subseteq V(G)$ such that $G-S$ contains more than $|S|$ nontrivial components.

If we apply Theorem 10 to our situation, then we observe that possibility (a) cannot occur. If there exists a cycle $C^{\prime}$ with $\left|V\left(C^{\prime}\right) \cap V(C)\right| \geq|V(C)|-1$, $\left|V\left(C^{\prime}\right) \cap V(H)\right| \geq 1$, and the vertices in $V\left(C^{\prime}\right) \cap V(H)$ form a path in $C^{\prime}$, then, by Claim 9, we can form a cycle $C_{0}$ with $\left|V\left(C_{0}\right) \cap V(C)\right| \geq$ $|V(C)|-1$ and $V(H) \subseteq V\left(C_{0}\right)$, hence $\left|V\left(C_{0}\right)\right| \geq n-1$, a contradiction. This proves the following claim.

Claim 10. There exists a nonempty subset $S \subseteq V(G)$ such that $G-S$ contains more than $|S|$ nontrivial components.

The 2-connected graphs $G$ on $n$ vertices with $\sigma_{3}(G) \geq n$ that satisfy the condition in Claim 10 are characterized in Bauer, Schmeichel, and Veldman [2]. Since the proof is rather short, we reproduce it here.

Let $S \subseteq V(G)$ be a nonempty cut set such that $G-S$ contains at least $|S|+1$ nontrivial components. Let $s=|S|$. Since $G$ is 2 -connected, we have $s \geq 2$. Let $H_{1}, H_{2}, \ldots, H_{s+1+j}(j \geq 0)$ be the nontrivial components of $G-S$ and let $n_{i}=\left|V\left(H_{i}\right)\right|(i=1, \ldots, s+1+j)$, where we assume $2 \leq n_{1} \leq n_{2} \leq \cdots \leq n_{s+1+j}$. Let $t$ be the number of trivial components of $G-S$.

By counting the number of neighbors of vertices in the three smallest components of $G-S$ we see

$$
n \leq \sigma_{3}(G) \leq n_{1}-1+n_{2}-1+n_{3}-1-\min \{3, t\}+3 s .
$$

Since $n=n_{1}+n_{2}+\ldots+n_{s+1+j}+s+t$, we obtain

$2(s+j-2) \leq n_{4}+\cdots+n_{s+1+j} \leq 2 s-t-\min \{3, t\}-3$,

implying that $2 j \leq 1-t-\min \{3, t\}$. We conclude that $j=t=0$.

If $s=2$, then we have $G \in \mathcal{F}_{2,1}(n)$.

If $s=3$, then (3) implies $n_{4} \leq 3$, hence $G \in \mathcal{F}_{2,2}(n)$.

Finally, if $s \geq 4$, then (3) implies $n_{s}=2$ and $n_{s+1} \leq 3$. If $n_{s+1}=3$, then $G \in \mathcal{F}_{2,3}(n)$, and if $n_{s+1}=2$, then $G \in \mathcal{F}_{2,4}(n)$.

This settles Case 4 and hence completes the proof of Theorem 6. 
Proof of Corollary 8. Let $G, C$, and $A$ be as defined in the statement of the corollary. Since none of the graphs in $\mathcal{F}(n)$ contains a dominating cycle, we have $c(G) \geq p(G)-1$, by Theorem 5 . Suppose $a, b \in(V(G)-$ $V(C)) \cup A^{+}$such that $a b \in E(G)$. Since $C$ is a dominating cycle, we can assume that $a \in A^{+}$and $b \in V(G)-V(C)$, or $a, b \in A^{+}$.

First suppose $a \in A^{+}$and $b \in V(G)-V(C)$. By the definition of $A$, there exist an $x_{a} \in V(G)-V(C)$ such that $a^{-} \in N\left(x_{a}\right)$. If $b=x_{a}$, then $b a \vec{C} a^{-} b$ is a cycle of length $|V(C)|+1$ contradicting the choice of $C$ as a longest cycle. And if $b \neq x_{a}$, then $b a \vec{C} a^{-} x_{a}$ is a path of length $|V(C)|+2$, contradicting $p(G) \leq c(G)+1$.

Next suppose $a, b \in A^{+}$, hence $a^{-}, b^{-} \in A$. There exist $x_{a}, x_{b} \in$ $V(G)-V(C)$ such that $a^{-} \in N\left(x_{a}\right)$ and $b^{-} \in N\left(x_{b}\right)$. Suppose $a$ and $b$ are neighbors on the cycle, say $b^{+}=a$. Then $x_{a} a^{-} \vec{C} b^{-} x_{b}$ is a cycle of length $|V(C)|+1$ (if $x_{a}=x_{b}$ ), or a path of length $|V(C)|+2$ (if $x_{a} \neq x_{b}$ ). In both cases we have a contradiction. So we can assume that $a$ and $b$ are not neighbors on the cycle. Then $x_{a} a^{-} \overleftarrow{C} b a \vec{C} b^{-} x_{b}$ is a cycle of length $|V(C)|+1$ (if $x_{a}=x_{b}$ ), or a path of length $|V(C)|+2$ (if $x_{a} \neq x_{b}$ ). So also in this last case, we always obtain a contradiction.

This shows that there exists no pair $a, b \in(V(G)-V(C)) \cup A^{+}$such that $a b \in E(G)$, thus proving Corollary 8 .

\section{References}

[1] D. Bauer, A. Morgana, E. F. Schmeichel, and H. J. Veldman, Long cycles in graphs with large degree sums. Discrete Math. 79 (1989/90) 59-70.

[2] D. Bauer, E. Schmeichel, and H.J. Veldman, A note on dominating cycles in 2-connected graphs. Preprint (1992).

[3] J. A. Bondy, Longest paths and cycles in graphs of high degree. Research Report CORR 80-16, Univ. of Waterloo, Waterloo, Ontario (1980).

[4] J.A. Bondy and U.S.R. Murty, Graph Theory with Applications. Macmillan, London and Elsevier, New York (1976).

[5] H. Enomoto, A. Kaneko, and Zs. Tuza, $P_{3}$-factors and covering cycles in graphs of minimum degree $n / 3$. A. Hajnal, L. Lovász, and V. T. Sós, editors, Combinatorics, Eger (Hungary), 1987. Colloquia Mathematica Societas János Bolyai 52. North-Holland, Amsterdam (1988) 213-219.

[6] J. van den Heuvel, Long cycles in graphs with large degree sums and neighborhood unions. Preprint (1993), J. Graph Theory; to appear.

[7] D. R. Woodall, The binding number of a graph and its Anderson number. J. Comb. Th. (B) 15 (1973) 225-255. 